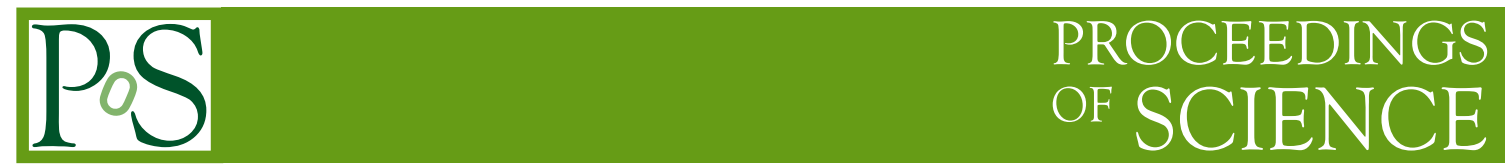

\title{
Radiative $B$ Decays at Belle
}

\author{
Akimasa Ishikawa* \\ Tohoku University \\ E-mail: akimasadepx.phys.tohoku.ac.ip
}

We report recent results on radiative $B$ decays at Belle at the KEKB collider.

The 39th International Conference on High Energy Physics (ICHEP 2018)

4-11 July, 2018

Seoul, Korea

${ }^{*}$ Speaker. 


\section{Introduction}

$B$ meson decays via loop diagrams are sensitive probe to new physics (NP). These processes in the SM are suppressed by the Cabibbo-Kobayashi-Maskawa matrix elements, $V_{t s}$ or $V_{t d}$, and a loop factor. Since unobserved heavy particles from some NP models can contributes the decays, the branching fractions, direct $C P$ violation $\left(A_{C P}\right)$, photon polarization, isospin asymmetry $\left(\Delta_{0+}\right.$ or $\left.\Delta_{0-}\right)$ and difference of $A_{C P}$ between charged and neutral $B$ mesons $\left(\Delta A_{C P}\right)$ might differ from the SM predictions. Radiative $B$ decays $b \rightarrow s \gamma$, which are mediated by loop diagrams, are experimentally and theoretically clean due to final states having a color singlet photon. Thus these are ideal tools to search for NP.

We report the measurements of radiative $B$ decays with a full data sample of $711 \mathrm{fb}^{-1}$ accumulated by the Belle detector at the KEKB energy-asymmetric collider.

\section{Evidence for Isospin Violation in $B \rightarrow K^{*} \gamma$ [U]}

We reconstruct $B^{0} \rightarrow K^{* 0} \gamma$ and $B^{+} \rightarrow K^{*+} \gamma$ decays, where $K^{*}$ is formed from $K^{+} \pi^{-}, K_{S}^{0} \pi^{0}$, $K^{+} \pi^{0}$ or $K_{S}^{0} \pi^{+}$combinations. To determine the branching fractions and direct $C P$ asymmetries as well as $\Delta A_{C P}$ and isospin asymmetry $\left(\Delta_{0+}\right)$, we perform extended unbinned maximum likelihood fits to the seven $M_{\mathrm{bc}}$ distributions and the results are

$$
\begin{aligned}
\mathscr{B}\left(B^{0} \rightarrow K^{* 0} \gamma\right) & =(3.96 \pm 0.07 \pm 0.14) \times 10^{-5}, \\
\mathscr{B}\left(B^{+} \rightarrow K^{*+} \gamma\right) & =(3.76 \pm 0.10 \pm 0.12) \times 10^{-5} \\
A_{C P}\left(B^{0} \rightarrow K^{* 0} \gamma\right) & =(-1.3 \pm 1.7 \pm 0.4) \% \\
A_{C P}\left(B^{+} \rightarrow K^{*+} \gamma\right) & =(+1.1 \pm 2.3 \pm 0.3) \% \\
A_{C P}\left(B \rightarrow K^{*} \gamma\right) & =(-0.4 \pm 1.4 \pm 0.3) \% \\
\Delta_{0+} & =(+6.2 \pm 1.5 \pm 0.6 \pm 1.2) \%, \\
\Delta A_{C P} & =(+2.4 \pm 2.8 \pm 0.5) \%,
\end{aligned}
$$

where the third uncertainty for $\Delta_{0+}$ is due to the uncertainty in $f_{+-} / f_{00}$. We find evidence for isospin violation in $B \rightarrow K^{*} \gamma$ decays with a significance of 3.1 $\sigma$, and this result is consistent with the predictions in the SM. Measured branching fractions and $A_{C P}$ are most precise to date.

\section{Measurement of Time-Dependent $C P$ Violation in $B \rightarrow K \eta \gamma$ [2]}

A measurement of time-dependent $C P$ violation in $B^{0} \rightarrow P_{1}^{0} P_{2}^{0} \gamma$ is the most promising method to measure the photon polarization in the $b \rightarrow s \gamma$ process, where $P_{1}^{0}$ and $P_{2}^{0}$ are scalar or pseudoscalar mesons and the $P_{1}^{0} P_{2}^{0}$ system is a $C P$ eigenstate. We measure the time-dependent $C P$ violation in $B^{0} \rightarrow K_{S}^{0} \eta \gamma$. We determine $C P$ violation parameters, $\mathscr{S}$ and $\mathscr{A}$, by performing an unbinned maximum-likelihood fit to the observed proper time difference distribution in the signal region. The obtained parameters

$$
\begin{aligned}
& \mathscr{S}=-1.32 \pm 0.77 \text { (stat.) } \pm 0.36 \text { (syst.), } \\
& \mathscr{A}=-0.48 \pm 0.41 \text { (stat.) } \pm 0.07 \text { (syst.) }
\end{aligned}
$$

are consistent with the null-asymmetry hypothesis within $2 \sigma$ as well as with SM predictions. 


\section{Measurements of Isospin Asymmetry and Difference of $C P$ Asymmetries in}

$$
B \rightarrow X_{s} \gamma[\text { [3] }
$$

Precision measurements of $B \rightarrow X_{s} \gamma$ branching fraction $\mathscr{B}\left(B \rightarrow X_{s} \gamma\right)$ [四, [] and direct $C P$

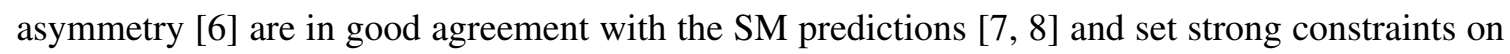
NP models. The theoretical uncertainties in the predictions of $\mathscr{B}\left(B \rightarrow X_{s} \gamma\right)$ and $A_{C P}\left(B \rightarrow X_{s} \gamma\right)$ are about $7 \%$ and $2 \%$ which are comparable with the experimental uncertainties of the current world averages [Q]. The Belle II experiment is expected to measure the branching fraction with a precision of about $3 \%[\mathbb{[}]$. Thus, the reduction of the theoretical uncertainty is crucial to further constrain NP models. One of the largest theoretical uncertainties is a resolved photon contribution which can be constrained from the measurement of isospin asymmetry in $B \rightarrow X_{s} \gamma$ [ए], ए2, [13]. A newly proposed $C P$ violation observable is a difference of the direct $C P$ asymmetries between the charged and neutral $B$ mesons, which is proportional to a ratio of Wilson coefficients $\operatorname{Im}\left(C_{8} / C_{7}\right)$, is null in the SM while in some NP models, this value could be as large as $10 \%$ [ [ 8 , 때, ㄷ]].

We perform measurements of $\Delta_{0-}$ and $\Delta A_{C P}$ in $B \rightarrow X_{s} \gamma$ with sum of 38 exclusive $X_{s}$ final states which cover about $77 \%$ of total $X_{s}$ decay rate. To extract the physics observables, we perform a simultaneous fit with an extended unbinned maximum likelihood method to eight $M_{\mathrm{bc}}$ distributions; five for $B^{-}, B^{+}, \bar{B}^{0}, B^{0}$, and $B_{\text {fns }}$ in the on-resonance data, and three for charged $B\left(B^{-}\right.$and $\left.B^{+}\right)$, flavor-specific neutral $B\left(\bar{B}^{0}\right.$ and $\left.B^{0}\right)$, and $B_{\mathrm{fns}}$ in the off-resonance data, where $B_{\mathrm{fns}}$ is denoted as flavor non-specific final states. The results are

$$
\begin{aligned}
\Delta_{0-} & =(-0.48 \pm 1.49 \pm 0.97 \pm 1.15) \%, \\
\Delta A_{C P} & =(+3.69 \pm 2.65 \pm 0.76) \% \\
A_{C P}^{\mathrm{C}} & =(+2.75 \pm 1.84 \pm 0.32) \% \\
A_{C P}^{\mathrm{N}} & =(-0.94 \pm 1.74 \pm 0.47) \% \\
A_{C P}^{\text {tot }} & =(+1.44 \pm 1.28 \pm 0.11) \% \\
\bar{A}_{C P} & =(+0.91 \pm 1.21 \pm 0.13) \%,
\end{aligned}
$$

where the third uncertainty for $\Delta_{0-}$ is due to $f_{+-} / f_{00}$. The measured $\Delta_{0-}$ and $\Delta A_{C P}$ are consistent with zero. Thus, these measurements can be used to constrain the resolved photon contribution in $B \rightarrow X_{s} \gamma$ and to constrain NP models. All the measurements are most precise to date.

\section{Summary}

We have measured the branching fractions, isospin asymmetries and $C P$ violation observables in radiative $B$ decays at Belle. These measurements strongly constrain NP models. Our measurements of $C P$ violation observables are dominated by the statistical uncertainty; thus, the upcoming Belle II experiment will further reduce the uncertainty. To improve the isospin asymmetry at Belle II, reduction of the dominant uncertainty due to $f_{+-} / f_{00}$ is essential, and can be performed at both Belle and Belle II.

\section{Acknowledgments}

A. I. is supported by the Japan Society for the Promotion of Science (JSPS) Grant No. 16H03968. 


\section{References}

[1] T. Horiguchi, A. Ishikawa et al. (Belle Collaboration), "Evidence for Isospin Violation and Measurement of $C P$ Asymmetries in $B \rightarrow K^{*}(892) \gamma$," Phys. Rev. Lett. 119, no. 19, 191802 (2017) doi:10.1103/PhysRevLett.119.191802 [arXiv:1707.00394 [hep-ex]].

[2] H. Nakano, A. Ishikawa et al. (Belle Collaboration), "Measurement of time-dependent $C P$ asymmetries in $B^{0} \rightarrow K_{S}^{0} \eta \gamma$ decays," Phys. Rev. D 97, no. 9, 092003 (2018) doi:10.1103/PhysRevD.97.092003 [arXiv:1803.07774 [hep-ex]].

[3] S. Watanuki, A. Ishikawa et al. (Belle Collaboration), "Measurements of isospin asymmetry and difference of direct $C P$ asymmetries in $B \rightarrow X_{s} \gamma$ decays with a sum-of-exclusive technique," arXiv:1807.04236 [hep-ex].

[4] A. Limosani et al. (Belle Collaboration), "Measurement of Inclusive Radiative $B$-meson Decays with a Photon Energy Threshold of 1.7-GeV,” Phys. Rev. Lett. 103, 241801 (2009) doi:10.1103/PhysRevLett.103.241801 [arXiv:0907.1384 [hep-ex]].

[5] T. Saito, A. Ishikawa et al. (Belle Collaboration), "Measurement of the $\bar{B} \rightarrow X_{s} \gamma$ Branching Fraction with a Sum of Exclusive Decays,” Phys. Rev. D 91, no. 5, 052004 (2015) doi:10.1103/PhysRevD.91.052004 [arXiv:1411.7198 [hep-ex]].

[6] S. Nishida et al. (Belle Collaboration), "Measurement of the CP asymmetry in $B \rightarrow X_{s} \gamma$," Phys. Rev. Lett. 93, 031803 (2004) doi:10.1103/PhysRevLett.93.031803 [hep-ex/0308038].

[7] M. Misiak et al., "Updated NNLO QCD predictions for the weak radiative B-meson decays," Phys. Rev. Lett. 114, no. 22, 221801 (2015) doi:10.1103/PhysRevLett.114.221801 [arXiv:1503.01789 [hep-ph]].

[8] M. Benzke, S. J. Lee, M. Neubert and G. Paz, "Long-Distance Dominance of the CP Asymmetry in $B \rightarrow X_{s, d} \gamma$ Decays," Phys. Rev. Lett. 106, 141801 (2011) doi:10.1103/PhysRevLett.106.141801 [arXiv:1012.3167 [hep-ph]].

[9] M. Tanabashi et al. (Particle Data Group), "Review of Particle Physics," Phys. Rev. D 98, no. 3, 030001 (2018). doi:10.1103/PhysRevD.98.030001

[10] E. Kou et al. (Belle II Collaboration), “The Belle II Physics Book,” arXiv:1808.10567 [hep-ex].

[11] S. J. Lee, M. Neubert and G. Paz, "Enhanced Non-local Power Corrections to the $\bar{B} \rightarrow X_{s} \gamma$ Decay Rate,” Phys. Rev. D 75, 114005 (2007) doi:10.1103/PhysRevD.75.114005 [hep-ph/0609224].

[12] M. Misiak, “ $\bar{B} \rightarrow X_{s} \gamma$ : Current Status,” Acta Phys. Polon. B 40, 2987 (2009) [arXiv:0911.1651 [hep-ph]].

[13] M. Benzke, S. J. Lee, M. Neubert and G. Paz, "Factorization at Subleading Power and Irreducible Uncertainties in $\bar{B} \rightarrow X_{s} \gamma$ Decay,” J. High Energy Phys. 08 (2010) 099 doi:10.1007/JHEP08(2010)099 [arXiv:1003.5012 [hep-ph]].

[14] R. Malm, M. Neubert and C. Schmell, "Impact of warped extra dimensions on the dipole coefficients in $\mathrm{b} \rightarrow \mathrm{s}$ Y transitions," J. High Energy Phys. 04 (2016) 042 doi:10.1007/JHEP04(2016)042 [arXiv:1509.02539 [hep-ph]].

[15] M. Endo, T. Goto, T. Kitahara, S. Mishima, D. Ueda and K. Yamamoto, “Gluino-mediated electroweak penguin with flavor-violating trilinear couplings,” J. High Energy Phys. 04 (2018) 019. doi:10.1007/JHEP04(2018)019 [arXiv:1712.04959 [hep-ph]]. 